\title{
A PBW theorem for inclusions of (sheaves of) Lie algebroids
}

\author{
Damien Calaque (*)
}

ABSTRACT - Inspired by the recent work of Chen-Stiénon-Xu on Atiyah classes associated to inclusions of Lie algebroids, we give a very simple criterium (in terms of those classes) for relative Poincaré-Birkhoff-Witt type results to hold. The tools we use (e.g. the first infinitesimal neighbourhood Lie algebroid) are straightforward generalizations of the ones previously developped by Căldăraru, Tu and the author for Lie algebra inclusions.

Mathematics Subject Classification (2010). 17B35, 16S30.

KEYwords. Lie algebroids, Atiyah class, PBW isomorphism.

\section{Contents}

1 - Introduction.................................. 24

1.1 - General context.............................. 24

1.2 - Description of the main results .................. 25

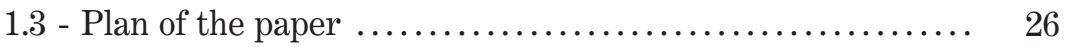

1.4 - Notation and conventions ........................ 26

2 - Lie algebroids and associated structures ............... 27

2.1 - The universal enveloping algebra of a Lie algebroid ..... 27

2.2 - The de Rham complex of a Lie algebroid .............. 29

2.3 - Lie algebroid jets ............................ 29

2.4 - Free Lie algebroids (after M. Kapranov) ............. 30

3 - Structures associated to inclusions of Lie algebroids ....... 31

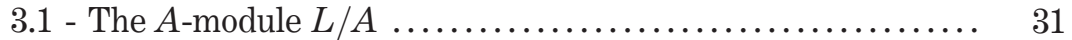

3.2 - The extension class $\alpha$ (inspired by Chen-Stiénon-Xu) .... 31

3.3 - The first infinitesimal neighbourhood Lie algebroid $A^{(1)} \ldots \quad 33$

(*) Indirizzo dell'A.: I3M, Université Montpellier 2, Case courrier 051, 34095 Montpellier cedex 5 - France.

E-mail: damien.calaque@univ-montp2.fr 
4 - PBW for the inclusion into the first infinitesimal neighbourhood 34

4.1 - Yet another look at the extension class $\alpha \ldots \ldots \ldots \ldots \ldots . \quad 35$

4.2 - A filtered morphism $j^{*} j_{!}\left(\mathbf{1}_{A}\right) \longrightarrow T_{R}(L / A) \ldots \ldots \ldots \ldots \ldots \ldots . \quad 36$

5 - PBW for an inclusion of Lie algebroids ................. 38

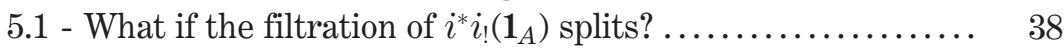

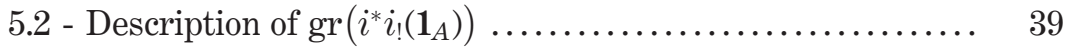

5.3 - What if $\alpha=0$ ? ................................ 39

6 - The case of an arbitrary $A$-module $E \ldots \ldots \ldots \ldots \ldots \ldots \ldots . . \ldots$

7 - Proof of the main Theorems (Theorems 1.1 and 1.2) and perspectives ......................................... 43

\section{Introduction}

\section{$1.1-$ General context}

This paper is part of a more general project which aims at building a dictionnary between Lie theory and algebraic geometry.

In [1] Arinkin and Căldăraru provide a necessary and sufficient condition for the Ext-algebra of a closed subvariety $X$ (a "brane") of an algebraic variety $Y$ to be isomorphic, as an object of the derived category of $X$, to $S(N[-1])$, where $N$ is the normal bundle of $X$ into $Y$; the condition is that $N$ can be lifted to the first infinitesimal neighbourhood $X^{(1)}$. This condition is equivalent to the vanishing of a certain class in $\operatorname{Ext}_{\mathcal{O}_{X}}^{2}\left(N^{\otimes 2}, N\right)$.

This result has been translated into Lie theory in [4] by Căldăraru, Tu and the author as follows. For an inclusion of Lie algebras $\mathfrak{h} \subset \mathfrak{g}$, we gave a necessary and sufficient condition for $U(\mathfrak{g}) / U(\mathfrak{g}) \mathfrak{h}$ to be isomorphic, as an $\mathfrak{h}$-module, to $S(\mathfrak{g} / \mathfrak{h})$; the condition is that the quotient module $\mathfrak{n}=\mathfrak{g} / \mathfrak{h}$ extends to a Lie algebra $\mathfrak{h}^{(1)}$ "sitting in between" $\mathfrak{h}$ and $\mathfrak{g}$. Similarly, this condition is equivalent to the vanishing of a certain class in $\operatorname{Ext}_{\mathfrak{f}}^{1}\left(\mathfrak{n}^{\otimes 2}, \mathfrak{n}\right)$.

It is Kapranov who observed in [9] that the shifted tangent bundle $T_{X}[-1]$ of an algebraic variety $X$ is a Lie algebra object in the derived category of $X$, with Lie bracket being given by the Atiyah class of $T_{X}[-1]$. Moreover, any object of the derived category becomes a representation of this Lie algebra via its own Atiyah class. In the case of a closed embedding $i: X \hookrightarrow Y$ we then get an inclusion of Lie algebras objects $T_{X}[-1] \subset i^{*} T_{Y}[-1]$, so that the main result of [1] can be deduced, in principle, from a version of the main result of [4] that would hold in a triangulated category. We refer to the introduction of [4] and to [3] for more details on this striking analogy. 
In [5] we have exhibited a Lie algebroid structure on the shifted normal bundle $N[-1]$ of a closed embedding $X \hookrightarrow Y$. Extending the results of [4] to the Lie algebroid setting then seems quite natural, especially if one wants to understand the geometry of a sequence of closed embeddings $X \hookrightarrow Y \hookrightarrow Z$.

\section{2 - Description of the main results}

For simplicity of exposition we assume in this introduction that $\mathbf{k}$ is a field of characteristic zero. We let $X$ be a topological space equipped with a sheaf of k-algebras $\mathcal{R}$, and $\mathcal{A} \subset \mathcal{L}$ be an inclusion of sheaves of Lie algebroids over $\mathcal{R}$ (we refer to Section 2 for standard Definitions), which are locally free as $\mathcal{R}$-modules. The locally free $\mathcal{R}$-module $\mathcal{L} / \mathcal{A}$ turns out to be naturally equipped with an action of $\mathcal{A}$ (see $\S 3.1$ ), also-known-as a flat $\mathcal{A}$ connection.

In [7] Chen, Stiénon and $\mathrm{Xu}$ introduce a very interesting class $\alpha_{\mathcal{E}} \in \operatorname{Ext}_{\mathcal{A}}^{1}\left((\mathcal{L} / \mathcal{A}) \otimes_{\mathcal{R}} \mathcal{E}, \mathcal{E}\right)$, for any $\mathcal{A}$-module $\mathcal{E}$, which is the obstruction to the existence of a lift of the flat $\mathcal{A}$-connection on $\mathcal{E}$ to a possibly non-flat $\mathcal{L}$ connection. They define this class in geometric terms, while we provide in this paper a purely algebraic description of $\alpha_{\mathcal{E}}$ (see $\S 3.2$ ) which makes sense in a wider context.

Inspired by a previous work [4] of the author with Căldăraru and Tu we also introduce a new Lie algebroid $A^{(1)}$, called the first infinitesimal neighbourhood Lie algebroid, which fits in between $\mathcal{A}$ and $\mathcal{L}$ in the sense that we have a sequence of Lie algebroid morphisms $\mathcal{A} \longrightarrow \mathcal{A}^{(1)} \longrightarrow \mathcal{L}$.

We then prove the following result, which generalizes [4, Theorem 1.3]:

THEOREM 1.1. The following statements are equivalent:

(1) The class $\alpha_{\mathcal{L} / \mathcal{A}}$ vanishes.

(2) The $\mathcal{A}$-module structure on $\mathcal{L} / \mathcal{A}$ lifts to an $A^{(1)}$-module structure.

(3) $U(\mathcal{L}) / U(\mathcal{L}) \mathcal{A}$ is isomorphic, as a filtered $\mathcal{A}$-module, to $S_{\mathcal{R}}(\mathcal{L} / \mathcal{A})$.

Here $S$ and $U$ denote the symmetric and the universal enveloping algebra, respectively.

We can also prove a more general version of the above result for $\mathcal{A}$ modules other than $\mathbf{1}_{\mathcal{A}}$ (compare with [4, Theorem 5.1]):

THEOREM 1.2. Let $\mathcal{E}$ be an $\mathcal{A}$-module which is locally free as an $\mathcal{R}$ module. Then the following statements are equivalent: 
(1) The classes $\alpha_{\mathcal{L} / \mathcal{A}}$ and $\alpha_{\mathcal{E}}$ vanish.

(2) The $\mathcal{A}$-module structures on $\mathcal{L} / \mathcal{A}$ and $\mathcal{E}$ lift to $A^{(1)}$-module structures.

(3) $U(\mathcal{L}) \otimes_{U(\mathcal{A})} \mathcal{E}$ is isomorphic, as a filtered $\mathcal{A}$-module, to $S_{\mathcal{R}}(\mathcal{L} / \mathcal{A}) \otimes_{\mathcal{R}} \mathcal{E}$.

\section{3 - Plan of the paper}

We start with some recollection about Lie algebroids in Section 2: e.g. we recall definitions and basic properties of the universal enveloping algebra, the de Rham (or Chevalley-Eilenberg) complex and jets of a given Lie algebroid, as well as the construction of free Lie algebroids. In Section 3 we introduce natural objects associated to an inclusion of Lie algebroids: the associated quotient module, the Chen-Stiénon-Xu class [7], and the first infinitesimal neighbourhood Lie algebroid. We also interprete the Chen-Stiénon-Xu class as the obstruction to extend modules to the first infinitesimal neighbourhood. Section 4 and 5 are the heart of the paper: the fourth Section is devoted to the statement and the proof of a PBW type theorem for inclusions into first infinitesimal neighbourhoods, from which we deduce the general case in the fifth Section. We then extend the previous results to general modules in Section 6 (this Section is to Sections 4 and 5 what Theorem 1.2 is to Theorem 1.1). We finally sheafify everything and prove the two Theorems of the Introduction in the last Section. We end the paper with an appendix where we prove that two classes coincide.

\section{4 - Notation and conventions}

Unless otherwise specified $\mathbf{k}$ is a commutative ring, all algebraic structures we consider are defined over $\mathbf{k}$, and all filtrations are ascending, indexed by non-negative integers. By an $n$-filtered morphism we mean a morphism that raises the filtration degree by $n$. A filtered morphism (a-ka morphism of filtered objects) is a 0-filtered morphism.

We now describe our conventions regarding tensor products. For a commutative ring $R$, we write $\otimes_{R}$ for the tensor product of left $R$-modules. As there is no ambiguity we define $\otimes:=\otimes_{\mathbf{k}}$. For a (possibly noncommutative) ring $B$, we denote by $\underset{B}{\otimes}$ the tensor product between right and left $B$-modules.

For a left $R$-module $M$ we denote by $S_{R}(M)$, resp. $T_{R}(M)$, the symmetric, resp. tensor, algebra generated by $M$ over $R$. Both are con- 
sidered as graded $R$-algebras; however, we don't require $R$ to be central in $R$-algebras. We write $S_{R}^{k}(M)$, resp. $T_{R}^{k}(M)$, for the $k$-the homogeneous component.

\section{Lie algebroids and associated structures}

Let $R$ be a commutative $\mathbf{k}$-algebra and $L$ a Lie algebroid over $R$, which means that the pair $(R, L)$ is a Lie-Rinehart algebra (see [12]). Namely, $L$ is a Lie k-algebra equipped with an $R$-module structure and an $R$-linear Lie algebra map $\rho: L \rightarrow \operatorname{Der}_{\mathbf{k}}(R)$ such that $\left[l, r l^{\prime}\right]=r\left[l, l^{\prime}\right]+\rho(l)(r) l^{\prime}$ for $l, l^{\prime} \in L$ and $r \in R$. The map $\rho$ is called the anchor map and we usually omit its symbol from the notation: for $l \in L$ and $r \in R$, we write $l(r):=\rho(l)(r)$. In particular $R \oplus L$ inherits the structure of a Lie k-algebra with bracket given by $\left[(r, l),\left(r^{\prime}, l^{\prime}\right)\right]=\left(l\left(r^{\prime}\right)-l^{\prime}(r),\left[l, l^{\prime}\right]\right)$, for $r, r^{\prime} \in R$ and $l, l^{\prime} \in L$.

What we discuss in this Section is relatively standard and can be found e.g. in $[12,13,10]$ and references therein (perhaps phrased in a different way).

\section{1 - The universal enveloping algebra of a Lie algebroid}

We define the enveloping algebra $U(R, L)$ of the pair $(R, L)$ to be the quotient of the positive part of the universal enveloping algebra ${ }^{1}$ of the Lie k-algebra $R \oplus L$ by the following relations: $r \otimes l=r l(r \in R, l \in R \oplus L)$. As there is no risk of confusion we simply write $U(L)$ for $U(R, L)$, which is obviously an $R$-algebra via the natural map $R \longrightarrow U(L)$. It therefore inherits an $R$-bimodule structure.

It turns out that $U(L)$ is also a cocommutative coring in left $R$-modules $^{2}$. Namely, the coproduct $\Delta: U(L) \longrightarrow U(L) \otimes_{R} U(L)$ is the multiplicative map defined on generators by $\Delta(r)=r \otimes 1=1 \otimes r(r \in R)$ and $\Delta(l)=l \otimes 1+1 \otimes l(l \in L)$. The anchor map can be extended to an $R$-al-

( $\left.{ }^{1}\right)$ By this we mean the subalgebra generated by $R \oplus L$ (i.e. the kernel of the natural k-augmentation).

$\left({ }^{2}\right)$ We would like to warn the reader that the multiplication is defined on $U(L) \otimes_{R} U(L)$ while the comultiplication takes values in $U(L) \otimes_{R} U(L)$, where only the left $R$-module structure is used. 
gebra morphism $U(L) \longrightarrow \operatorname{End}(R)$ (actually taking values in the ring $\operatorname{Diff}(R)$ of differential operators) sending $r \in R$ to the multiplication by $r$ and $l \in L$ to $\rho(l)$. The counit $\varepsilon: U(L) \longrightarrow R$ is defined by $\varepsilon(P):=P(1)$.

REMARK 2.1. The above definition of $\Delta$ needs some explanation. Being the quotient of $U(L) \otimes U(L)$ by the right ideal generated by $r \otimes 1-1 \otimes r$ $(r \in R), U(L) \otimes_{R} U(L)$ is not an algebra. Nevertheless, one easily sees that $r \otimes 1(r \in R)$ and $l \otimes 1+1 \otimes l(l \in L)$ sit in the normalizer of that ideal, so that multiplying them together makes perfect sense.

In what follows, left $U(L)$-modules are called $L$-modules. We say that a given (left) $R$-module $E$ is acted on by $L$ if it is equipped with an $L$ module structure of which the restriction to $R$ gives back the original $R$ action we started with. The abelian category $L$-mod of $L$-modules is monoidal, with product being $\otimes_{R}$ (and $U(L)$ acting on a tensor product via the coproduct) and unit $\mathbf{1}_{L}$ being $R$ equipped with the action given by the anchor $\rho$.

Any morphism $f: L \longrightarrow L^{\prime}$ of Lie algebroids over $R$ automatically induces a morphism of algebras $U(L) \longrightarrow U\left(L^{\prime}\right)$ which preserves all the above algebraic structures. We denote the restriction (or pull-back) functor $L^{\prime}$-mod $\longrightarrow L$-mod by $f^{*}$, and by $f_{!}:=U\left(L^{\prime}\right) \underset{U(L)}{\otimes}$ - its left adjoint. Notice that $f^{*}$ is monoidal, while $f !$ is not ( $f_{\text {! }}$ is only colax-monoidal).

There is a canonical filtration on $U(L)$ obtained by assigning degree 0 , resp. 1 , to elements of $R$, resp. $L$. All structures we have defined so far on $U(L)$ respect this filtration. If, additionally, $L$ is itself equipped with a filtration, then this filtration extends to $U(L)$. The canonical filtration on $U(L)$ can be seen as coming from the obvious "constant" filtration on $L$ (the only degree 0 element is 0 and all elements in $L$ are of degree $\leq 1)$.

REMARK 2.2. One can alternatively describe the functor $U$ as a left adjoint. Namely, we consider the category of anchored algebras: they are defined as $R$-algebras $B$ equipped with an $R$-algebra morphism $\rho: B \longrightarrow \operatorname{End}(R)$, where the $R$-algebra structure on $\operatorname{End}(R)$ is the given by $r \longmapsto\left(l_{r}: b \mapsto r b\right)$. There is a functor Prim from anchored algebras to Lie algebroids that sends an anchored algebra $B$ to the sub- $R$-module consisting of those elements $b \in B$ such that $\rho(b) \in \operatorname{Der}(R)$. We then have an adjuntion

$$
U:\{\text { Lie algebroids }\} \rightleftarrows\{\text { anchored algebras }\}: \text { Prim . }
$$




\section{2 - The de Rham complex of a Lie algebroid}

To any $L$-module $E$ we associate the complex of graded $R$-modules $C^{\bullet}(L, E)$, consisting of $\operatorname{Hom}_{R}\left(\wedge_{R}^{\bullet} L, E\right)$ equipped with the differential $d$ defined as follows: for $\omega \in C^{n}(L, E)$ and $l_{0}, \ldots, l_{n} \in L$,

$$
\begin{aligned}
d(\omega)\left(l_{0}, \ldots, l_{n}\right):=\sum_{i=0}^{n}(-1)^{i} l_{i} \omega\left(l_{0}, \ldots, \widehat{l_{i}}, \ldots, l_{n}\right)+ \\
\quad \sum_{i<j}(-1)^{i+j} \omega\left(\left[l_{i}, l_{j}\right], l_{0}, \ldots, \widehat{l_{i}}, \ldots, \widehat{l_{j}}, \ldots, l_{n}\right) .
\end{aligned}
$$

The map that associates to $l \in L$ the element $\nabla_{l} \in \operatorname{End}_{\mathbf{k}}(E)$ defined by $\nabla_{l}(e):=d(e)(l)$ is sometimes called a flat connection. It completely determines both the differential $d$ and the $L$-action on $E$.

We have the following functoriality property: for $f: L \rightarrow L^{\prime}$ a morphism of Lie algebroids over $R$ and $\varphi: E \rightarrow F$ a morphism of $L^{\prime}$-modules, we have an obvious $R$-linear map $f^{*} \varphi: C^{\bullet}\left(L^{\prime}, E\right) \longrightarrow C^{\bullet}\left(L, f^{*} F\right)$ defined by $\left(f^{*} \varphi\right)(\omega):=\varphi \circ \omega \circ f$. We also have that for any two $L$-modules $E$ and $F$, there is a product $C^{\bullet}(L, E) \otimes C^{\bullet}(L, F) \longrightarrow C^{\bullet}\left(L, E \otimes_{R} F\right)$. In particular, this turns $C^{\bullet}(L):=C^{\bullet}\left(L, \mathbf{1}_{L}\right)$ into a differential graded commutative $R$ algebra.

\section{3 - Lie algebroid jets}

For any $L$-module $E$ we define the $L$-module $J_{L}(E)$ of $L$-jets, or simply jets, as the internal Hom $\operatorname{Hom}_{R}(U(L), E)$ from the universal enveloping algebra $U(L)$ to $E$.

This requires some explanation. First of all observe that the monoidal category $L$-mod is closed. The internal Hom of two $L$-modules $E$ and $F$ is given by the $R$-module $\operatorname{Hom}_{R}(E, F)$ equipped with the following $L$-action: for $l \in L, \psi: E \rightarrow F$ and $e \in E,(l \cdot \psi)(e):=l \cdot(\psi(e))-$ $\psi(l \cdot e)$. Then $U(L)$ is naturally an $L$-module (being a left $U(L)$-module over itself).

But $U(L)$ is actually an $U(L)$-bimodule. Therefore, $J_{L}(E)$ inherits a second left $U(L)$-module structure, denoted $*$, which commutes with the above one and is defined in the following way: for $\phi \in J_{L}(E)$ and $P, Q \in U(L),(P * \phi)(Q)=\phi(Q P)$. When $E=\mathbf{1}_{L}$ the two commuting $L$ module structures one gets on $J_{L}:=J_{L}\left(\mathbf{1}_{E}\right)$ are precisely the ones described in [6, § 4.2.5]. 
REMARK 2.3. This is actually true for any $U(L)$ - $U\left(L^{\prime}\right)$-bimodule $M$ : the space $\operatorname{Hom}_{R}(M, E)$ has an $L$-module and an $L^{\prime}$-module structures that commute $^{3}$. In particular, the space $\operatorname{Hom}_{L-\bmod }(M, E)$ itself is naturally an $L^{\prime}$-module.

\section{4 - Free Lie algebroids (after M. Kapranov)}

Let us first recall from [10] that an $R$-module $M$ is anchored if it is equipped with an $R$-linear map $\rho: M \longrightarrow \operatorname{Der}(R)$, called the anchor map. Like for Lie algebroids we usually omit the symbol $\rho$ from our notation: for $m \in M$ and $r \in R$, we write $m(r):=\rho(m)(r)$. There is an obvious forgetful functor which goes from the category of Lie algebroids to that of anchored modules, that forgets everything except the underlying $R$-module structure of the Lie algebroid and the anchor map. This functor has a left adjoint, denoted $F R$.

For any anchored $R$-module $M$ we call $F R(M)$ the free Lie algebroid generated by $M$. It can be described in the following way, as a filtered quotient of the free Lie k-algebra $F L(M)$ generated by $M$. First of all, by adjunction $\rho$ naturally extends to a Lie k-algebra morphism $F L(M) \longrightarrow \operatorname{Der}(R)$. Then we define $F R(M)$ as the quotient of $F L(M)$ by the following relations: for $r \in R, m \in F L(M)$, and $m^{\prime} \in F L(M), \quad\left[m, r m^{\prime}\right]-\left[r m, m^{\prime}\right]=m(r) m^{\prime}+m^{\prime}(r) m$. These relations being satisfied in $\operatorname{Der}(R)$ then $\rho$ factors through $F R(M)$. Finally, we define an $R$-module structure on $F R(M)$ in the following way: $r\left[m, m^{\prime}\right]:=\left[m, r m^{\prime}\right]-m(r) m^{\prime}=\left[r m, m^{\prime}\right]+m^{\prime}(r) m$.

According to Remark 2.2 we then have a sequence of adjunctions

\{anchored modules $\} \stackrel{F R}{\rightleftarrows}\{$ Lie algebroids $\} \underset{\text { Prim }}{\stackrel{U}{\rightleftarrows}}\{$ anchored algebras $\}$.

REMARK 2.4. The above sequence of adjunctions extends to filtered versions. Unless otherwise specified, the canonical filtration we put on an anchored module $M$ is the "constant" one we already mentioned in $\S 2.1$. Then the associated graded of the induced filtration on $F R(M)$ is the free Lie $R$-algebra $F L_{R}(M)$ generated by $M$, and the associated graded of the induced filtration on $U(F R(M))$ is $U\left(F L_{R}(M)\right)=T_{R}(M)$.

$\left({ }^{3}\right)$ Notice that even the two underlying $R$-module structures are different. 


\section{Structures associated to inclusions of Lie algebroids}

Let $R$ be a commutative $\mathbf{k}$-algebra and $i: A \hookrightarrow L$ an inclusion of Lie algebroids over $R$.

\section{1 - The A-module $L / A$}

It is well-known that $A$ does not necessarily act on itself (meaning that $A$ is not an $A$-module in any natural way). In this paragraph we consider the quotient $R$-module $L / A$ and define an $A$-action on it in the following way: for any $a \in A$ and any $l \in L$, we define $a \cdot(l+A):=[a, l]+A$ (when there is no ambiguity we omit the inclusion symbol $i$ from the notation). The only nontrivial identity to check is that $(r a) \cdot=r(a \cdot)$ :

$(r a) \cdot(l+A)=[r a, l]+A=r[a, l]-\underbrace{l(r) a}_{\in A}+A=r[a, l]+A=r(a \cdot(l+A))$.

From now and in the rest of the paper we make the following assumption:

The $\operatorname{map} L \longrightarrow U(L)$ is injective.

\section{2 - The extension class $\alpha$ (inspired by Chen-Stiénon-Xu)}

Let $E$ be an $A$-module. We define a class $\alpha_{E} \in \operatorname{Ext}_{A}^{1}\left((L / A) \otimes_{R} E, E\right)$, which generalizes the one introduced in [4] for Lie algebras, via the following short exact sequence of $A$-modules:

$$
0 \longrightarrow E \longrightarrow(U(L) \underset{U(A)}{\otimes} E)^{\leq 1} \longrightarrow L / A \otimes_{R} E \longrightarrow 0 .
$$

We have to explain why the middle term in (1) is an $A$-module, which is a priori not guaranteed. Namely, even though $U(L)$ is an $A$-module (via left multiplication) its filtered pieces $U(L)^{\leq k}$ are not (because $\left.A U(L)^{\leq k} \subset U(L)^{\leq k+1}\right)$. Nevertheless, $U(L) \otimes E$ turns out to be a filtered $A$-module because of the following: for $a \in A, P \in U(L)^{\leq k}$ and $e \in E$,

(2) $a(P \otimes e)=a P \otimes e=([a, P]+P a) \otimes e=$

$$
[a, P] \otimes e+P \otimes a e \in(U(L) \underset{U(A)}{\otimes} E)^{\leq k} .
$$

We set $\alpha:=\alpha_{L / A}$. 
Relation to Atiyah classes as they are defined by Chen-Stiénon-Xu in [7]

We consider the filtered subspace $J_{L / A}(E)$ of $J_{L}(E)$ consisting of those maps $\phi: U(L) \rightarrow E$ which are $A$-linear: for $Q \in U(A)$ and $P \in U(L)$,

$$
\phi(Q P)=Q \cdot \phi(P) .
$$

According to Remark 2.3 there is a residual $L$-module structure on $J_{L / A}(E)$ : for $Q \in U(L)$, we have $(Q * \phi)(P)=\phi(P Q)$. Even though the successive quotients $J_{L}^{n}(E):=\operatorname{Hom}_{R}\left(U(L)^{\leq n}, E\right)$ of $J_{L}(E)$ are not $U(L)$-bimodules, it turns out that their subspaces $J_{L / A}^{n}(E)$ inherits an $A$-action from the above residual $L$-action. Namely, for $Q \in U(A), \phi \in J_{L / A}(E)$ and $P \in U(L)$ we have

$$
(Q * \phi)(P)=\phi(P Q)=\phi([P, Q]+Q P)=\phi([P, Q])+Q \cdot(\phi(P)) .
$$

Therefore $Q *$ descends to $J_{L / A}^{n}(E)$. We then have the following exact sequence of $A$-modules:

$$
0 \longrightarrow \operatorname{Hom}_{R}(L / A, E) \longrightarrow J_{L / A}^{1}(E) \longrightarrow E \longrightarrow 0 .
$$

This determines a class $\widetilde{\alpha}_{E} \in \operatorname{Ext}_{A}^{1}\left(E, \operatorname{Hom}_{R}(A, E)\right)$, which has been first defined in a differential geometric context by Chen-Stiénon-Xu in $[7, \S 2.5 .1]$.

Proposition 3.1. The images of the classes $\alpha_{E}$ and $\widetilde{\alpha}_{E}$ coincide in

$$
\operatorname{Hom}_{D(A)}\left((L / A) \stackrel{\mathrm{L}}{\otimes_{R}} E, E[1]\right) \cong \operatorname{Hom}_{D(A)}\left(E, \mathbb{R} \operatorname{Hom}_{R}(L / A, E)[1]\right),
$$

where $D(A)$ denotes the bounded derived category of A-modules.

Proof. See Appendix.

In particular this implies that $\alpha_{E}=0$ if and only if $\widetilde{\alpha}_{E}=0$ (which we also prove in Proposition 3.3). This is because the natural maps from $\operatorname{Ext}_{A}^{1}\left((L / A) \otimes_{R} E, E\right)$ and $\operatorname{Ext}_{A}^{1}\left(E, \operatorname{Hom}_{R}(L / A, E)\right)$ to $\operatorname{Hom}_{D(A)}\left((L / A) \stackrel{\mathrm{L}}{\otimes}_{R} E, E[1]\right)$ and $\operatorname{Hom}_{D(A)}\left(E, \operatorname{R}_{\operatorname{Hom}}(L / A, E)[1]\right)$, respectively, are both injective.

REMARK 3.2. It is very likely that $\tilde{\alpha}_{E}$ fits into the framework of Atiyah classes associated to dDg algebras [6, Section 8], but proving it would require to understand deeply the Koszul-type duality between $C^{\bullet}(L)$ and $U(L)$ (see e.g. [11] in which the two extreme cases $L=\operatorname{Der}(R)$ and $R=\mathbf{k}$ are covered). 


\section{3 - The first infinitesimal neighbourhood Lie algebroid $A^{(1)}$}

Being a Lie algebroid over $R, L$ is in particular an anchored $R$-module. We can therefore consider the free Lie algebroid $F R(L)$ over $R$ generated by $L$. Let us then consider the quotient $A^{(1)}$ of $F R(L)$ by the ideal generated by ${ }^{4}$

$$
[a, l]_{F R(L)}-[a, l]_{L}, \quad a \in A, l \in L .
$$

Observe that it is a well-defined (Lie algebroid) ideal in $F R(L)$ as the anchor map of $F R(L)$ coincides by definition with the one of $L$ on generators. We call $A^{(1)}$ the first infinitesimal neighbourhood of $A$ (see [4], where the geometric motivation behind such a denomination is given). We denote by $j$ the Lie algebroid inclusion of $A$ into $A^{(1)}$. We now prove that, for an $A$ module $E$, the classes $\alpha_{E}$ and $\widetilde{\alpha}_{E}$ both give the obstruction to lift $E$ to an $A^{(1)}{ }_{-}$ module:

Proposition 3.3. Let $E$ be an A-module. Then the following statements are equivalent:

(1) There exists an $A^{(1)}$-module $E^{(1)}$ such that $j^{*}\left(E^{(1)}\right)=E$.

(2) $\alpha_{E}=0$.

(3) $\widetilde{\alpha}_{E}=0$.

From now we omit the symbol · in formulæ. We also omit parentheses when they are not strictly necessary (e.g. when one can use associativity).

Proof. (1) $\Longrightarrow(2)$ : assume that such an $A^{(1)}$-module $E^{(1)}$ exists. Observe that any $P \in U^{\leq 1}(L)=R \oplus L$ lies in $U(F R(L))$, and thus acts on $E$ through the quotient map $U(F R(L)) \rightarrow U\left(A^{(1)}\right)$. We therefore set, for any $e \in E, s(P \otimes e):=P e$. Since $E=j^{*}\left(E^{(1)}\right)$ then there is no ambiguity in the way $Q \in U(A)$ acts on $E$, so that $s(P Q \otimes e)=P Q e=s(P \otimes Q e)$. Moreover, for the same reason $s$ is $A$-linear:

$$
Q s(P \otimes e)=Q P e=[Q, P]_{A^{(1)}} e+P Q e=[Q, P]_{L} e+P Q e=s(Q(P \otimes e)) .
$$

Therefore $s$ is a splitting of (1).

$(2) \Longrightarrow(3)$ : assume $\alpha_{E}=0$. Then there exists a splitting $s$ : $(U(L) \underset{U(A)}{\otimes} E)^{\leq 1} \longrightarrow E$ of (1). We define a splitting $\widetilde{s}: E \longrightarrow J_{L / A}^{1} E$ of (5)

$\left({ }^{4}\right)$ Observe that, contrary to what is suggested by the notation, $A^{(1)}$ does not only depend on $A$ but also on $L$. 
as follows: for $P \in U(L)^{\leq 1}$ and $e \in E, \widetilde{s}(e)(P):=s(P \otimes e)$. We must check two things:

- $\widetilde{s}(e)$ belongs to $J_{L / A}(E)$, i.e. satisfies (3): if $Q \in U(A)$ then

$$
\widetilde{s}(e)(Q P)=s(Q P \otimes e)=Q(s(P \otimes e))=Q(\widetilde{s}(e)(P)) .
$$

- $\widetilde{s}$ is $A$-linear: if $Q \in U(A)$ then $(Q * \widetilde{s}(e))(P)=\widetilde{s}(e)(P Q)=s(P Q \otimes e)=$ $s(P \otimes Q e)=\widetilde{s}(Q e)(P)$.

(3) $\Longrightarrow$ (1): finally assume we have a section $s: E \rightarrow J_{L / A}(E)$ of (5). Then for any $l \in L$ and any $e \in E$ we define $l e:=s(e)(l)$. We first observe that this defines an action of $F R(L)$ on $E$ :

- on the one hand if $r \in R, l \in L, e \in E$ then $(r l) e=s(e)(r l)=$ $r(s(e)(l))=r(l e)$.

- on the other hand if $r \in R, l \in L, e \in E$ then

$l(r e)-r(l e)=s(r e)(l)-s(e)(r l)=$

$$
(r * s(e))(l)-s(e)(r l)=s(e)(l r-r l)=s(e)(l(r))=l(r) e .
$$

This action restricts to the one of $A \subset F R(L)$ : for $a \in A$ and $e \in E$, $s(e)(a)=a s(e)(1)=a e$. Finally we see that it descends to an action of $A^{(1)}$ : for $a \in A, l \in L$ and $e \in E$,

$$
\begin{aligned}
& \left([l, a]_{L}\right) e=s(e)\left([l, a]_{L}\right)= \\
& \quad(a * s(e))(l)-a(s(e)(l))=(s(a e))(l)-a l e=l a e-a l e=\left([l, a]_{F R(L)}\right) e .
\end{aligned}
$$

In the second equality we have used formula (4) for the $A$-action on $J_{L / A}(E)$ and in the third one we have used $A$-linearity of $s$.

\section{PBW for the inclusion into the first infinitesimal neighbourhood}

In this Section we prove a version of the main Theorem for the inclusion $j: A \hookrightarrow A^{(1)}$. The proof we give follows very much and hopefully simplifies the one of Darij Grinberg for Lie algebras (see [8]). It is very likely that a proof using some Koszulness property in the spirit of [4] might also exist ${ }^{5}$.

$\left({ }^{5}\right)$ But it would have required to adapt some standard but quite technical constructions to the context of non-central $R$-algebras (see Remark 3.2). 
The goal of the present Section is to understand the $A$-module

$$
j^{*} j_{!}\left(\mathbf{1}_{A}\right):=U\left(A^{(1)}\right) \underset{U(A)}{\otimes} \mathbf{1}_{A}=U\left(A^{(1)}\right) / U\left(A^{(1)}\right) A .
$$

According to $\S 2.4$ the free Lie algebroid $F R(L)$ and its quotient $A^{(1)}$ admit a filtration. Their universal enveloping algebras are therefore filtered too in an obvious way. We denote these filtrations by $F^{k} U(F R(L))$ and $F^{k} U\left(A^{(1)}\right)$ in order to distinguish them from the standard filtrations on universal enveloping algebras. It is worth noticing that the $A$-module structure on $j^{*} j_{!}\left(\mathbf{1}_{A}\right)$ is compatible with the induced filtration $F^{k}:=F^{k}\left(j^{*} j_{!}\left(\mathbf{1}_{A}\right)\right)$ : for any $a \in A$ and any $P \in F^{k} U\left(A^{(1)}\right)$, we have

$$
\begin{aligned}
& a\left(P+U\left(A^{(1)}\right) A\right)= \\
& a P+U\left(A^{(1)}\right) A=[a, P]+U\left(A^{(1)}\right) A \subset F^{k} U\left(A^{(1)}\right)+U\left(A^{(1)}\right) A .
\end{aligned}
$$

According to $\S 2.4$ the associated graded algebra of the filtered $R$-algebra $U(F R(L))$ is the tensor $R$-algebra $T_{R}(L)$. The filtered $R$-linear surjection $\xi: U(F R(L)) \rightarrow j^{*} j_{!}\left(\mathbf{1}_{A}\right)$ therefore induces a graded $R$-linear map $\operatorname{gr}(\xi): T_{R}(L) \rightarrow \operatorname{gr}\left(j^{*} j_{!}\left(\mathbf{1}_{A}\right)\right)$. We shall also use the graded $R$-algebra surjection $\pi: T_{R}(L) \rightarrow T_{R}(L / A)$.

THEOREM 4.1. The class $\alpha=\alpha_{L / A}$ vanishes if and only if there exists an isomorphism of filtered A-modules $\varphi: j^{*} j_{!}\left(\mathbf{1}_{A}\right) \longrightarrow T_{R}(L / A)$ such that $\operatorname{gr}(\varphi \circ \xi)=\pi$. Moreover, when this happens one can choose $\varphi$ so that it is $A^{(1)}$-linear.

We devote the rest of this Section to the proof of Theorem 4.1.

\section{1 - Yet another look at the extension class $\alpha$}

The sequence of filtered Lie algebroids morphism ${ }^{6} A \longrightarrow A^{(1)} \longrightarrow L$ provides us with a morphism of filtered $U(A)$-algebras $U\left(A^{(1)}\right) \longrightarrow U(L)$. It turns out to restrict to an isomorphism (of $R$-modules) $F^{1} U\left(A^{(1)}\right) \stackrel{\sim}{\longrightarrow} U(L)^{\leq 1}$ between the first filtered pieces.

$\left({ }^{6}\right)$ Here the filtration put on $A$, resp. $L$, is the constant one, so that the induced filtration on its universal enveloping algebra is the standard one. 
Therefore we get the following isomorphism of extensions (meaning that the diagram commutes and the lines are exact sequences), for any $A$ module $E$ :

(6)

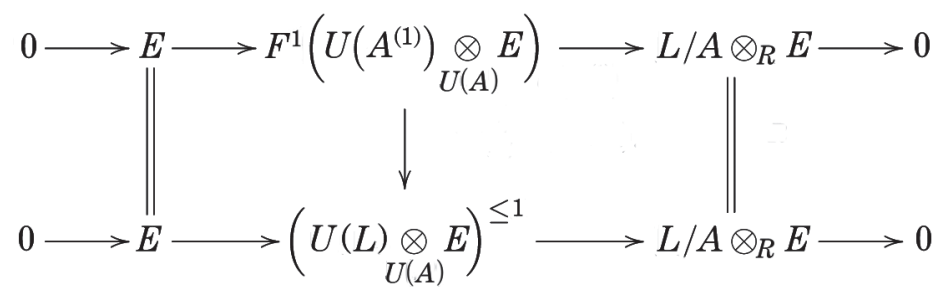

Let us now restrict our attention to the case when $E=L / A$. We have a 1filtered $A$-module morphism $\psi: U\left(A^{(1)}\right) \underset{U(A)}{\otimes}(L / A) \longrightarrow U^{+}\left(A^{(1)}\right) / U^{+}\left(A^{(1)}\right) A$, where $U^{+}\left(A^{(1)}\right)=\operatorname{ker}(\varepsilon) \cong U\left(A^{(1)}\right) / R$ is equipped with the induced filtration. Notice that $U^{+}\left(A^{(1)}\right) / U^{+}\left(A^{(1)}\right) A \cong j^{*} j_{!}\left(\mathbf{1}_{A}\right) / F^{0}$.

We also have an identification $F^{1} / F^{0}=L / A$ so that the following diagram of $A$-modules commutes (again, here lines are exact):

(7)

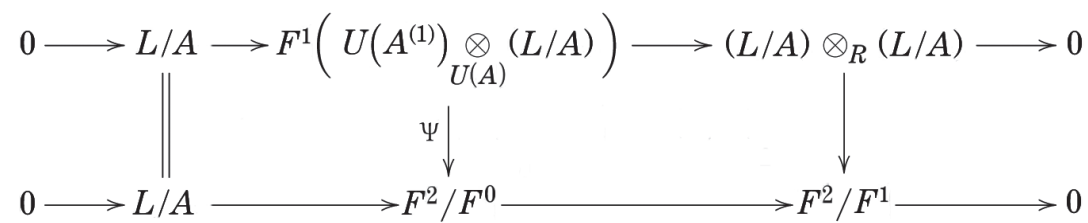

We can now prove the "if" part of Theorem 4.1.

Proposition 4.2. If the filtration of $j^{*} j_{!}\left(\mathbf{1}_{A}\right)$ splits, then $\alpha=0$.

Proof. If the filtration of $j^{*} j_{!}\left(\mathbf{1}_{A}\right)$ splits, then the bottom exact sequence in the diagram (7) splits and therefore so does the top exact sequence in the same diagram. It follows from the commutativity of (6) that the class of this exact sequence is $\alpha=\alpha_{L / A}$.

\section{$4.2-$ A filtered morphism $j^{*} j_{!}\left(\mathbf{1}_{A}\right) \longrightarrow T_{R}(L / A)$}

We now assume that $\alpha=0$, which means that the $A$-action on $L / A$ can be lifted to an $A^{(1)}$-action. We therefore obtain a graded $A^{(1)}$-module structure on $T_{R}(L / A)$. We use the notation - for this action. For any $l \in L$ and any $P \in T_{R}(L / A)$ we now define $l \bullet P:=l \cdot P+\bar{l} \otimes P, \bar{l}$ being the class of $l$ in $L / A$. 
LEMMA 4.3. The operation • defines a filtered $F R(L)$-module structure on $T_{R}(L / A)$ such that:

(i) It actually is an $A^{(1)}$-module structure.

(ii) Its restriction to $A$ is the original $A$-module structure on $T_{R}(L / A)$.

Proof. First of all let us prove that $\bullet$ determines an $F R(L)$-action on $T_{R}(L / A)$ : for $r \in R$ and $l \in L$ we obviously have $(r l) \bullet-=r(l \bullet-)$, and for any $P \in T_{R}(L / A)$,

$r(l \bullet P)-l \bullet(r P)=r l \cdot P+r \bar{l} \otimes P-l \cdot(r P)-\bar{l} \otimes r P=r l \cdot P-l \cdot(r P)=l(r) P$.

We now prove that it turns out to be an $A^{(1)}$-action: for $a \in A, l \in L$ and $P \in T_{R}(L / A)$

$$
\begin{aligned}
{[a, l]_{F R(L)} \bullet P } & =a \bullet(l \bullet P)-l \bullet(a \bullet P)=[a, l]_{A^{(1)}} \cdot P+a \cdot(\bar{l} \otimes P)-\bar{l} \otimes(a \cdot P) \\
& =[a, l]_{L} \cdot P+(a \cdot \bar{l}) \otimes P=[a, l]_{L} \cdot P+\overline{[a, l]_{L}} \otimes P=[a, l]_{L} \bullet P .
\end{aligned}
$$

Finally, the second property is obvious.

We obtain from the above lemma a filtered morphism of $A^{(1)}$-modules

$$
\varphi: U\left(A^{(1)}\right) / U\left(A^{(1)}\right) A \longrightarrow T_{R}(L / A), \quad P \longmapsto P \bullet 1 .
$$

It is clear from the construction of $\varphi$ that $\operatorname{gr}(\varphi \circ \xi)=\pi$, which is surjective. Therefore $\operatorname{gr}(\varphi)$ is surjective, and thus $\varphi$ is surjective (because filtrations under consideration are exhaustive).

We will now prove that $\varphi$ is an isomorphism. To do so we will prove that $\operatorname{gr}(\varphi)$ is an isomorphism. We start with the following:

Lemma 4.4. The two-sided ideal $\langle A\rangle$ generated by $A$ in $T_{R}(L)$ sits inside the kernel of $\operatorname{gr}(\xi)$.

Proof. Recall that the kernel of $\xi: U(F R(L)) \longrightarrow U\left(A^{(1)}\right) / U\left(A^{(1)}\right) A=$ $j^{*} j_{!}\left(\mathbf{1}_{A}\right)$ is the sum of the two-sided ideal generated by $[a, l]_{F R(L)}-[a, l]_{L}$ ( $a \in A, l \in L$ ) and of the left ideal generated by $A$. In particular, for $a \in A$, $l \in L, P \in F^{k} U(F R(L))$ and $Q \in F^{l} U(F R(L))$, we have:

$$
P(a l-l a) Q \in \operatorname{ker}(\xi)+F^{k+l+1} U(F R(L)) \text { and } P a \in \operatorname{ker}(\xi) .
$$

Therefore the two-sided ideal generated by $a \otimes l-l \otimes a(a \in A, l \in L)$ 
in $T_{R}(L)$ sits inside $\operatorname{ker}(\operatorname{gr}(\xi))$, as well as does the left ideal generated by $A$. Together they generate the two-sided ideal generated by $A$ inside $T_{R}(L)$.

It follows from the above lemma that $\operatorname{gr}(\xi)$ induces a surjective map $T_{R}(L) /\langle A\rangle \longrightarrow \operatorname{gr}\left(j^{*} j_{!}\left(\mathbf{1}_{A}\right)\right)$ such that, when composed with the surjective map $\operatorname{gr}(\varphi): \operatorname{gr}\left(j^{*} j_{!}\left(\mathbf{1}_{A}\right)\right) \longrightarrow T_{R}(L / A)$, it leads to the isomorphism $T_{R}(L) /\langle A\rangle \longrightarrow T_{R}(L / A)$. In particular, $\operatorname{gr}(\varphi)$ is an isomorphism.

\section{PBW for an inclusion of Lie algebroids}

The goal of the present Section is to understand the $A$-module

$$
i^{*} i_{!}\left(\mathbf{1}_{A}\right):=U(L) \underset{U(A)}{\otimes} \mathbf{1}_{A}=U(L) / U(L) A .
$$

According to $\S 3.2 i^{*} i_{!}\left(\mathbf{1}_{A}\right)$ turns out to be a filtered $A$-module. We write $G^{k}:=\left(i^{*} i_{!}\left(\mathbf{1}_{A}\right)\right)^{\leq k}$, and borrow the notation from the previous Section.

$5.1-$ What if the filtration of $i^{*} i_{!}\left(\mathbf{1}_{A}\right)$ splits?

Proposition 5.1. If the filtration of $i^{*} i_{!}\left(\mathbf{1}_{A}\right)$ splits, then $\alpha=0$.

Proof. We have a 1-filtered $A$-module morphism $\psi: U(L) \underset{U(A)}{\otimes}(L / A) \longrightarrow$ $U^{+}(L) / U^{+}(L) A$, where $U^{+}(L)=\operatorname{ker}(\varepsilon) \cong U(L) / R$ is equipped with the induced filtration.

We then have the following commuting diagram, in which lines are exact:

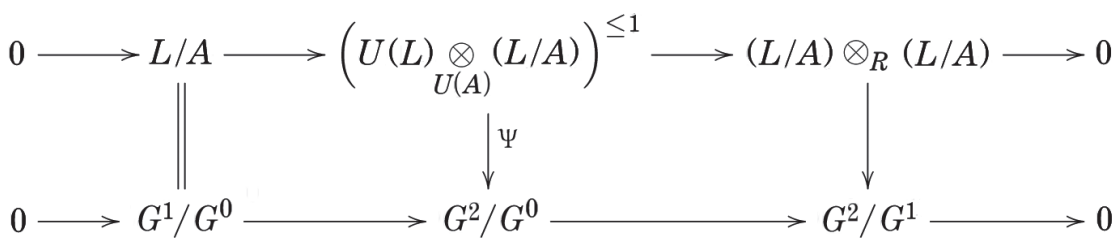

Finally, if the filtration of $i^{*} i_{!}\left(\mathbf{1}_{A}\right)$ splits then the bottom line in the above diagram splits too, and therefore so does the top one (of which the extension class is precisely $\alpha=\alpha_{L / A}$ ). 


\section{2 - Description of $\operatorname{gr}\left(i^{*} i_{!}\left(\mathbf{1}_{A}\right)\right)$}

We have the following coCartesian square of filtered (res. graded) $R$-modules:

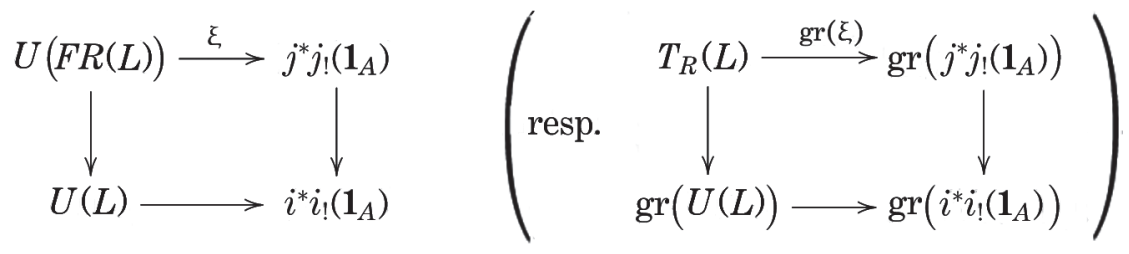

We have already seen (Lemma 4.4) that the associated graded of $\xi: U(F R(L)) \longrightarrow j^{*} j_{!}\left(\mathbf{1}_{A}\right)$ descends to a surjective map $T_{R}(L) /\langle A\rangle \longrightarrow$ $\operatorname{gr}\left(j^{*} j_{!}\left(\mathbf{1}_{A}\right)\right)$. In a completely similar way one can prove that associated graded of the maps $U(F R(L)) \longrightarrow U(L)$ and $U(F R(L)) \longrightarrow i^{*} i_{!}\left(\mathbf{1}_{A}\right)$ descend to surjective maps $\left.S_{R}(L) \longrightarrow \operatorname{gr}(U(L))\right)$ and $S_{R}(L) /\langle A\rangle \longrightarrow \operatorname{gr}\left(i^{*} i_{!}\left(\mathbf{1}_{A}\right)\right)$.

Let us assume that the following two properties hold:

(*) The surjective R-linear map $S_{R}(L) \longrightarrow \operatorname{gr}(U(L))$ is an isomorphism.

( $\left.\star^{\prime}\right)$ The surjective $R$-linear map $T_{R}(L) /\langle A\rangle \longrightarrow \operatorname{gr}\left(j^{*} j_{!}\left(\mathbf{1}_{A}\right)\right)$ is an isomorphism.

Remark 5.2. Property ( $\star$ ) is known to hold if $L$ is a projective $R$ module (see [12]). Property ( $\star$ ) also implies Assumption $(\varnothing)$ that we've made from $\S 3.1$. Thanks to the results of the previous Section, Property $\left(\star^{\prime}\right.$ ) follows from the assumption that $\alpha=0$.

Then coCartesianity of the diagram

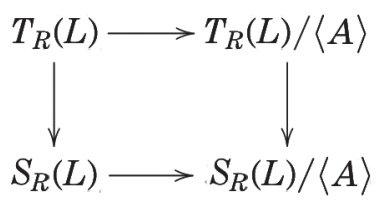

ensures us that the surjective $R$-linear map $S_{R}(L) /\langle A\rangle \longrightarrow \operatorname{gr}\left(i^{*} i_{!}\left(\mathbf{1}_{A}\right)\right)$ is an isomorphism.

\section{$5.3-$ What if $\alpha=0$ ?}

In this paragraph we consider the following property:

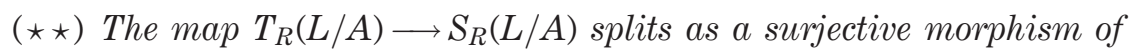
graded A-modules. 
Notice that the symmetrization map $S_{R}(L / A) \longrightarrow T_{R}(L / A)$ provides a splitting whenever $Q \subset R$. If we assume that $(\star \star)$ and $\alpha=0$ hold, then we have a sequence

$$
S_{R}(L / A) \hookrightarrow T_{R}(L / A) \cong j^{*} j_{!}\left(\mathbf{1}_{A}\right) \rightarrow i^{*} i_{!}\left(\mathbf{1}_{A}\right)
$$

of filtered $A$-module morphisms.

Lemma 5.3. Passing to associated graded in (9) we get exactly the surjective map $S_{R}(L) /\langle A\rangle \longrightarrow \operatorname{gr}\left(i^{*} i_{!}\left(\mathbf{1}_{A}\right)\right)$ that appears in $\S 5.2$.

Proof. It follows from the commutativity of the diagram

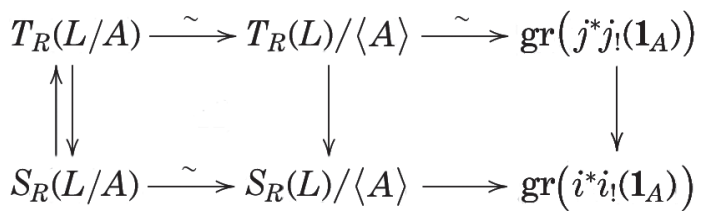

where the leftmost (upward) arrow is the same as the leftmost arrow in (9) (i.e. a given splitting of $T_{R}(L / A) \rightarrow S_{R}(L / A)$ ).

If we further assume that Property $(\star)$ holds, then we have proved in $\S 5.2$ that this map is an isomorphism (remember that $\alpha=0$ implies $\left(\star^{\prime}\right)$ ). Therefore, we have:

Theorem 5.4. Assume Properties ( $\star$ ) and $(\star \star)$ hold. Then $\alpha=0$ if and only if there exists an isomorphism of filtered A-modules $S_{R}(L / A) \longrightarrow i^{*} i_{!}\left(\mathbf{1}_{A}\right)$.

\section{The case of an arbitrary $A$-module $E$}

Let now $E$ be an $A$-module, and consider the following two $A$-modules:

$$
j^{*} j_{!}(E):=U\left(A^{(1)}\right) \underset{U(A)}{\otimes} E \quad \text { and } \quad i^{*} i_{!}(E):=U(L) \underset{U(A)}{\otimes} E .
$$

We denote by $F_{E}^{n}$ and $G_{E}^{n}$ the filtration pieces on those two filtered $A$ modules ${ }^{7}$. One sees that

${ }^{(7)}$ Even though the filtered pieces of $U\left(A^{(1)}\right)$ and $U(L)$ are not $A$-modules, $F_{E}^{n}$ and $G_{E}^{n}$ are. Namely, for any $a \in A$ and any $P \otimes e$ in $F_{E}^{n}$ (resp. $G_{E}^{n}$ ), $a(P \otimes e)=a P \otimes e=([a, P]-P a) \otimes e=[a, P] \otimes e-P \otimes a e \in F_{E}^{n}$ (resp. $\left.G_{E}^{n}\right)$. 


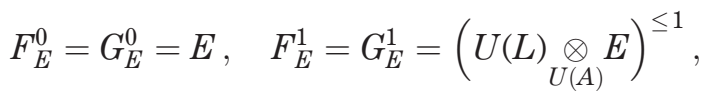

$$
\begin{aligned}
& \text { and } F_{E}^{1} / F_{E}^{0}=G_{E}^{1} / G_{E}^{0}=(L / A) \otimes_{R} E \text {. }
\end{aligned}
$$

Therefore, if the filtration on either $j^{*} j_{!}(E)$ or $i^{*} i_{!}(E)$ splits then $\alpha_{E}=0$. We start with the following generalization of Theorem 4.1:

THEOREM 6.1. Assume that $E$ is faithful ${ }^{8}$. Then both classes $\alpha$ and $\alpha_{E}$ vanish if and only if there exists an isomorphism filtered A-modules $\varphi_{E}: j^{*} j_{!}(E) \longrightarrow T_{R}(L / A) \otimes_{R} E$ such that $\left.\operatorname{gr}\left(\varphi_{E} \circ \xi_{E}\right)\right)=\pi_{E}$.

Here $\xi_{E}: U(F R(L)) \otimes_{R} E \stackrel{\xi \otimes \operatorname{id}_{E}}{\longrightarrow} U\left(A^{(1)}\right) \underset{U(A)}{\otimes} E \quad$ and $\quad \pi_{E}: T_{R}(L) \otimes_{R} E \stackrel{\pi \otimes \mathrm{id}_{E}}{\longrightarrow}$ $T_{R}(L / A) \otimes_{R} E$.

Sketch of Proof. From the above we can assume that $\alpha_{E}=0$, which means that the $A$-module $E$ lifts to an $A^{(1)}$-module $E^{(1)}$. This allows one to construct a surjective filtered morphism of $A^{(1)}$-modules

$\eta_{E}: j_{!}(E) \longrightarrow j_{!}\left(\mathbf{1}_{A}\right) \otimes_{R} E^{(1)} ; P \otimes e \longmapsto P \cdot(1 \otimes e), \quad\left(P \in U\left(A^{(1)}\right)\right.$ and $\left.e \in E\right)$.

It is well-defined: for any $a \in A,(P a) \cdot(1 \otimes e)=P \cdot(a \otimes e+1 \otimes a e)=$ $P \cdot(1 \otimes a e)$.

We now prove an analog of Proposition 4.2 in $\S 4.1$. We have the following commutative diagram of $A$-modules in which lines are exact:

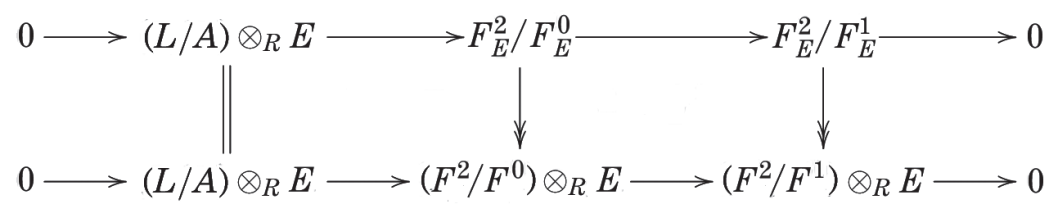

If the filtration $\left(F_{E}^{n}\right)_{n \geq 0}$ splits (in $A$-mod) then so does the top line in the above diagram, and thus the bottom line splits too (this is because the rightmost vertical arrow in (10) is surjective). Faithfulness of $E$ ensures that $0 \rightarrow F^{1} / F^{0} \rightarrow F^{2} / F^{0} \rightarrow F^{2} / F^{1} \rightarrow 0$ splits, which implies that $\alpha=0$ (see $\S 4.1$ ).

$\left({ }^{8}\right)$ Here we mean that $E$ is faithful as an $R$-module, which ensures that $-\otimes_{R} E: A$-mod $\rightarrow A$-mod is faithful (because the forgetful functor $A$-mod $\rightarrow$ $R$-mod is) and thus reflects exact sequences. 
Conversely, if we assume that $\alpha=0$ then by Theorem 4.1 we get a surjective morphism of filtered $A$-modules $\varphi_{E}:=\left(\varphi \otimes \operatorname{id}_{E}\right) \circ j^{*} \eta_{E}: j^{*} j_{!}(E) \longrightarrow$ $T_{R}(L / A) \otimes_{R} E$. We show it is an isomorphism by proving it on the level of associated graded. One can see that $\langle A\rangle_{R} E$ lies in the kernel of $\operatorname{gr}\left(\xi_{E}\right)$. To conclude, one just observes that on associated graded the composed surjection

$$
\left(T_{R}(L) /\langle A\rangle\right) \otimes_{R} E \stackrel{\operatorname{gr}\left(\xi_{E}\right)}{\longrightarrow} j^{*} j !(E) \stackrel{\operatorname{gr}\left(\varphi_{E}\right)}{\longrightarrow} T_{R}(L / A) \otimes_{R} E
$$

coincide with the standard surjection $\left(T_{R}(L) /\langle A\rangle\right) \otimes_{R} E \rightarrow T_{R}(L / A) \otimes_{R} E$, which is an isomorphism.

We now generalize Theorem 5.4:

Theorem 6.2. Assume that $E$ is faithful and that Properties ( $\star$ ) and $(\star \star)$ hold. Then $\alpha$ and $\alpha_{E}$ both vanish if and only if there exists an isomorphism of filtered $A$-module $S_{R}(L / A) \otimes_{R} E \longrightarrow i^{*} i_{!}(E)$.

Sketch of Proof. As before we can assume that $\alpha_{E}=0$, so that there exists an $A^{(1)}$-module $E^{(1)}$ such that $j^{*} E^{(1)}=E$. In particular, as we have seen in the proof of Theorem 6.1, there exists a surjective morphism of filtered $A$-modules $j^{*} \eta_{E}: j^{*} j_{!}(E) \longrightarrow j^{*} j_{!}\left(\mathbf{1}_{A}\right) \otimes_{R} E$.

We first go through the analog of Proposition 5.1 in $\S 5.1$. We have the following commutative diagram of $A$-modules in which lines are exact:

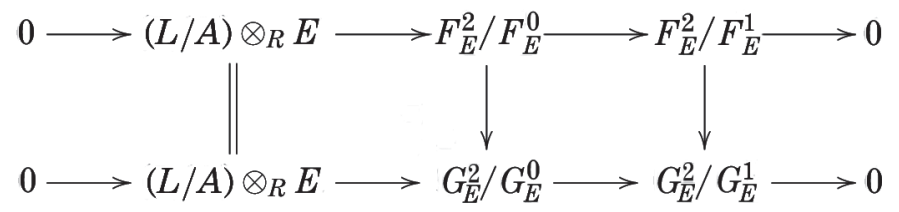

If the filtration of $i^{*} i_{!}(E)$ splits then so does the bottom line in diagram (11), and thus the top line in the same diagram splits too. Therefore $\alpha=0$ (see the proof of Theorem 6.1).

Let us now assume that $\alpha=0$. Then we have seen in the proof of Theorem 6.1 that $j^{*} \eta_{E}$ is an isomorphism of filtered $A$-modules.

Lemma 6.3. $\operatorname{gr}\left(j^{*} \eta_{E}\right)$ descends to an A-module isomorphism $\operatorname{gr}\left(i^{*} i_{!}(E)\right) \stackrel{\sim}{\longrightarrow} \operatorname{gr}\left(i^{*} i_{!}\left(\mathbf{1}_{A}\right) \otimes_{R} E\right)$.

Proof OF the Lemma. We have two coCartesian diagrams of filtered $R$-modules 

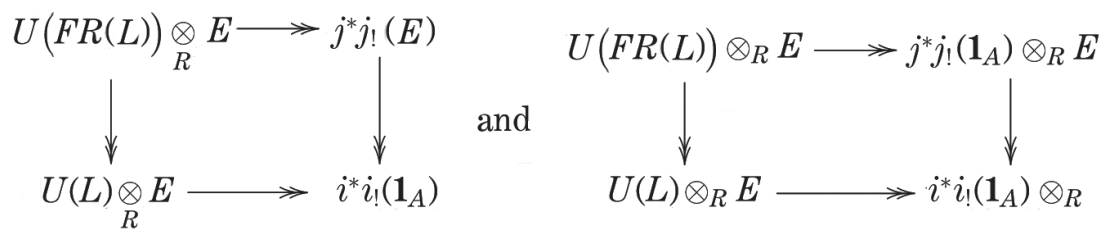

Passing to associated graded $R$-modules we get the following commutative diagram in which squares are coCartesian:

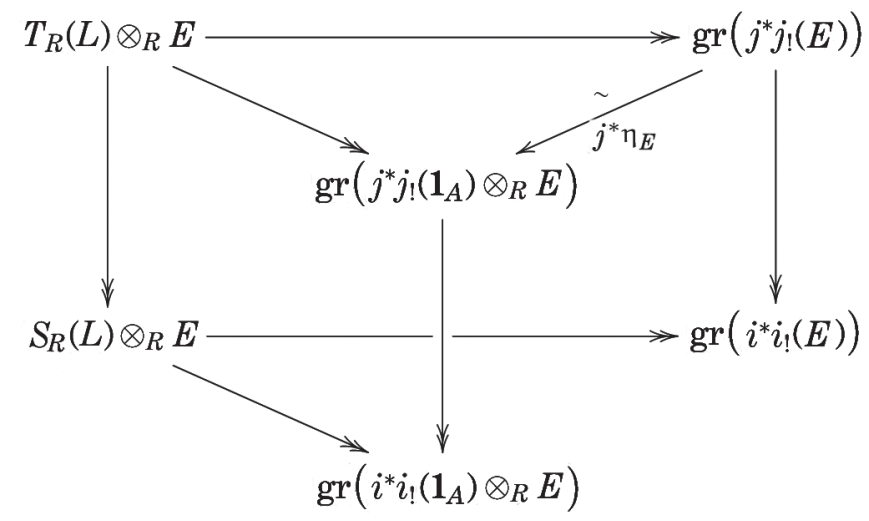

Therefore we get an $R$-linear isomorphism $\operatorname{gr}\left(i^{*} i_{!}(E)\right) \stackrel{\sim}{\longrightarrow}$ $\operatorname{gr}\left(i^{*} i_{!}\left(\mathbf{1}_{A}\right) \otimes_{R} E\right)$, which happens to be $A$-linear (this is because the square it files is made of surjective $A$-linear maps).

In particular, applying $-\otimes_{R} E$ to the diagram (8) ensures that the surjective morphism of graded $R$-modules $S_{R}(L / A) \otimes_{R} E \cong$ $\left(S_{R}(L) /\langle A\rangle\right) \otimes_{R} E \rightarrow \operatorname{gr}\left(i^{*} i_{!}(E)\right)$ is an isomorphism. We finally have a sequence of filtered morphisms of $A$-modules

$$
S_{R}(L / A) \otimes_{R} E \hookrightarrow T_{R}(L / A) \otimes_{R} E \stackrel{\varphi_{E}^{-1}}{\longrightarrow} j^{*} j_{!}(E) \rightarrow i^{*} i_{!}(E),
$$

for which one can check that the associated graded is the above isomorphism $S_{R}(L / A) \otimes_{R} E \stackrel{\sim}{\longrightarrow} \operatorname{gr}\left(i^{*} i_{!}(E)\right)$.

\section{Proof of the main Theorems (Theorems 1.1 and 1.2) and perspectives}

All what we have done so far can be sheafified. I.e. everything remains true if we replace $\mathbf{k}$ by a sheaf of rings $\mathcal{K}, R$ by a sheaf of $\mathcal{K}$-algebras $\mathcal{R}$, 
$i: A \hookrightarrow L$ by an inclusion of sheaves of Lie algebroids $i: \mathcal{A} \hookrightarrow \mathcal{L}$ over $\mathcal{R}$, and $E$ by an $\mathcal{A}$-module $\mathcal{E}$.

We now deduce Theorem 1.1 from the sheafified version of Theorem 5.4.

Proof of Theorem 1.1. From now $\mathcal{K}=\underline{\mathbf{k}}_{X}$ and $\mathbf{k}$ is a field of zero characteristic. The equivalence between (1) and (2) is the sheafified version of Proposition 3.3.

Assume now that $\mathcal{L}$ is locally free as an $\mathcal{R}$-modules so that, after [12], Property $(\star)$ holds. Finally, $\mathbf{k}$ being of zero characteristic we can take the symmetrization map $S_{\mathcal{R}}(\mathcal{L} / \mathcal{A}) \longrightarrow T_{\mathcal{R}}(\mathcal{L} / \mathcal{A})$ as a splitting of $T_{\mathcal{R}}(\mathcal{L} / \mathcal{A}) \longrightarrow$ $S_{\mathcal{R}}(\mathcal{L} / \mathcal{A})$ in $\mathcal{A}$-modules. Therefore Property $(\star \star)$ holds.

We apply (the sheafified version of) Theorem 5.4 to get the desired result.

Similarly, we deduce Theorem 1.2 from (the sheafified version of) Theorem 6.2 (one just has to notice that locally free modules are faithful).

\section{Perspectives}

Let $\imath: X \hookrightarrow Y$ be a closed embeddings of smooth algebraic varieties. It is shown in [5] (see also [14]) that the shifted normal bundle $\mathcal{A}=N_{X / Y}[-1]$ is a Lie algebroid object in the derived category $D\left(\underline{\mathbf{k}}_{X}\right)$ of sheaves of $\mathbf{k}$ modules on $X$. Namely, the anchor map $N_{X / Y}[-1] \longrightarrow T_{X}$ is given by the normal bundle exact sequence

$$
0 \longrightarrow T_{X} \longrightarrow \imath^{*} T_{Y} \longrightarrow N_{X / Y} \longrightarrow 0
$$

and the Lie bracket comes from the fact that $N_{X / Y}[-1]$ is the cohomology of the relative tangent complex ${ }^{\top} T_{X / Y}$. Moreover, it is proved in [5] that the universal enveloping algebra of this Lie algebroid is $\imath^{*} \imath_{1} \mathcal{O}_{\mathcal{X}}$. According to [1] it satisfies the PBW condition ( $\star$ ) if and only if a certain class in $\operatorname{Ext}_{\mathcal{O}_{\mathcal{X}}}^{2}\left(\wedge^{2}\left(N_{X / Y}\right), N_{X / Y}\right)$ vanishes. It can be understood as the class of the following extention:

$$
0 \longrightarrow N_{X / Y}[-1] \longrightarrow U^{+}(\mathcal{A})^{\leq 2} \longrightarrow S^{2}\left(N_{X / Y}[-1]\right) \longrightarrow 0 .
$$

Now let $\jmath: Y \hookrightarrow Z$ be another closed embedding of smooth algebraic varieties and consider the Lie algebroid object $\mathcal{L}=N_{X / Z}[-1]$ in $D(X)$. We have a Lie algebroid inclusion $\mathcal{A} \rightarrow \mathcal{L}$, with $\mathcal{L} / \mathcal{A}=\imath^{*} N_{Y / Z}[-1]$. It would be interesting to understand the geometric meaning of our main result in this context, and its relation to the sequence of derived self-intersections $X \times_{Y}^{h} X \rightarrow X \times \times_{Z}^{h} X \rightarrow Y \times{ }_{Z}^{h} Y$. 
It would also be interesting to search for a geometric interpretation of our main result in the direction of what Bordemann did for the Lie algebra case in [2].

\section{Appendix: sketch of proof of Proposition 3.1}

We borrow the notation from $\S 3.2$ and start with the following:

Lemma 7.1. The A-module $J_{L / A}^{1}(E)$ is isomorphic to the kernel of the difference map

$$
E \oplus \operatorname{Hom}_{R}\left(L / A,(U(L) \underset{U(A)}{\otimes} E)^{\leq 1}\right) \longrightarrow \operatorname{Hom}_{R}\left(L / A, L / A \otimes_{R} E\right) .
$$

Sketch of Proof. We first construct a map $\ell: J_{L / A}^{1}(E) \longrightarrow$ $\operatorname{Hom}_{R}\left(L / A,(U(L) \underset{U(A)}{\otimes} E)^{\leq 1}\right)$; for any $\phi \in J_{L / A}^{1}(E)$ and any $l \in L$ we set $\ell(\phi)(l):=l \otimes \phi(1)-1 \otimes \phi(l) \in(U(L) \otimes E E)^{\leq 1}$. First of all observe that for any $a \in A, \ell(\phi)(a)=a \otimes \phi(1)-1 \otimes \phi(a)=a \otimes \phi(1)-1 \otimes a \phi(1)=0$. Hence $\ell(\phi)$ factors through $L / A$. Then we show that $\ell$ is $A$-linear: for $\phi \in J_{L / A}^{1}(E)$, $a \in A$, and $l \in L$,

$$
\begin{aligned}
(a \cdot \ell(\phi))(l) & =a \cdot(\ell(\phi)(l))-\ell(\phi)([a, l]) \\
& =a l \otimes \phi(1)-a \otimes \phi(l)-[a, l] \otimes \phi(1)+1 \otimes \phi([a, l]) \\
& =l a \otimes \phi(1)-a \otimes \phi(l)+1 \otimes \phi([a, l]) \\
& =l \otimes \phi(a)-1 \otimes \phi(l a) \\
& =\ell(a * \phi)(l) .
\end{aligned}
$$

Finally, composing $\ell(\phi)$ with the epimorphism $(U(L) \underset{U(A)}{\otimes} E)^{\leq 1} \longrightarrow L / A \otimes_{R} E$ we get $l \mapsto l \otimes \phi(1)$, which coincides with the image of $\phi$ through $J_{L / A}^{1}(E) \rightarrow$ $E \rightarrow \operatorname{Hom}_{R}\left(L / A, L / A \otimes_{R} E\right)$. This provides a morphism from $J_{L / A}^{1}(E)$ to the kernel of the difference map (12).

We now construct an inverse to that map. For any $e+f$ in the kernel of (12), where $e \in E$ and $f \in \operatorname{Hom}_{R}\left(L / A,(U(L) \underset{U(A)}{\otimes} E)^{\leq 1}\right)$, we associate an element $\phi_{e, f}$ of $J_{L / A}^{1}(E)$ in the following way. For any $r \in R$ we set $\phi_{e, f}(r):=r e$. If $l \in L$ then one notices that $f(l)-l \otimes e \in(U(L) \underset{U(A)}{\otimes} E)^{\leq 1}$ projects onto zero in $L / A \otimes_{R} E$ and thus has the form $1 \otimes \phi_{e, f}(l)$. 
We now turn to the proof Proposition 3.1. Let us assume that we have an extension

$$
0 \longrightarrow E \longrightarrow B \longrightarrow(L / A) \otimes_{R} E \longrightarrow 0
$$

representing a class $\beta \in \operatorname{Ext}_{A}^{1}\left((L / A) \otimes_{R} E, E\right) \cong \operatorname{Hom}_{D(A)}\left((L / A) \otimes_{R} E, E[1]\right)$. We would like to describe the image of $\beta$ through the map

$$
\begin{aligned}
\operatorname{Hom}_{D(A)}\left((L / A) \otimes_{R} E, E[1]\right) \longrightarrow & \operatorname{Hom}_{D(A)}\left((L / A) L \otimes_{R} E, E[1]\right) \cong \\
& \operatorname{Hom}_{D(A)}(E, \mathbb{R} \operatorname{Hom}(L / A, E[1])) .
\end{aligned}
$$

One first chooses an injective resolution $\widetilde{E}$ of $E$ and considers the induced exact sequence of complexes

$$
0 \longrightarrow \widetilde{E} \longrightarrow \widetilde{B} \longrightarrow(L / A) \otimes_{R} \widetilde{E} \longrightarrow 0,
$$

where $\widetilde{B}$ is the cokernel of $E \longrightarrow \widetilde{E} \oplus B$; one then applies $\operatorname{Hom}_{A}(L / A,-)$ and get an exact sequence

$0 \longrightarrow \operatorname{Hom}_{A}(L / A, \widetilde{E}) \longrightarrow \operatorname{Hom}_{A}(L / A, \widetilde{B}) \longrightarrow \operatorname{Hom}_{A}\left(L / A,(L / A) \otimes_{R} \widetilde{E}\right) \longrightarrow 0$.

One finally gets an exact sequence

$$
0 \longrightarrow \operatorname{Hom}_{A}(L / A, \widetilde{E}) \longrightarrow \widetilde{C} \longrightarrow \widetilde{E} \longrightarrow 0,
$$

where $\widetilde{C}$ is the kernel of $\widetilde{E} \oplus \operatorname{Hom}_{A}(L / A, \widetilde{B}) \longrightarrow \operatorname{Hom}_{A}\left(L / A,(L / A) \otimes_{R} \widetilde{E}\right)$. This defines the desired element in $\operatorname{Hom}_{D(A)}(E, \mathbb{R} \operatorname{Hom}(L / A) E,[1]) \cong$ $\operatorname{Hom}_{D(A)}(\widetilde{E}, \operatorname{Hom}(L / A, \widetilde{E}[1]))$.

Proof of Proposition. We apply the above construction to $\beta=\alpha_{E}$, with $B=(U(L) \underset{U(A)}{\otimes} E)^{\leq 1}$. One first easily sees that $\widetilde{B}=(\underset{U(A)}{U(L) \underset{E}{\otimes}})^{\leq 1}$. Then it follows from Lemma 7.1 that $\widetilde{C}=J_{L / A}^{1}(\widetilde{E})$. Therefore, the image of $\alpha_{E}$ through the map (13) is determined by the exact sequence

$$
0 \longrightarrow \operatorname{Hom}_{A}(L / A, \widetilde{E}) \longrightarrow J_{L / A}^{1}(\widetilde{E}) \longrightarrow \widetilde{E} \longrightarrow 0,
$$

which is precisely the image of the class $\widetilde{\alpha}_{E} \in \operatorname{Ext}_{A}^{1}(E, \operatorname{Hom}(L / A, E))$ through the map

$$
\begin{aligned}
& \operatorname{Ext}_{A}^{1}(E, \operatorname{Hom}(L / A, E)) \cong \operatorname{Hom}_{D(A)}(E, \operatorname{Hom}(L / A, E)[1]) \longrightarrow \\
& \operatorname{Hom}_{D(A)}(E, \mathbb{R} \operatorname{Hom}(L / A, E)[1]) .
\end{aligned}
$$

The Proposition is proved. 
Acknowledgments. I thank Darij Grinberg for his careful reading of a preliminary version of this paper. His comments helped me correct some mistakes and improve the exposition. I also thank the anonymous referee for her/his extremely valuable suggestions. This project has been partially supported by a grant from the Swiss National Science Foundation (project number 200021_137778).

\section{REFERENCES}

[1] D. Arinkin, A. CĂLdĂRARU, When is the self-intersection of a subvariety a fibration?, Advances in Math., 231 (2012), no. 2, 815-842.

[2] M. Bordemann, Atiyah classes and equivariant connections on homogeneous spaces, Travaux Mathématiques, 20 (2012), Special Issue dedicated to Nikolai Neumaier, 29-82.

[3] D. Calaque, From Lie theory to algebraic geometry and back, in Interactions between Algebraic Geometry and Noncommutative Algebra, Oberwolfach Report no. 22/2010, 1331-1334.

[4] D. Calaque, A. Căldăraru, J. Tu, PBW for an inclusion of Lie algebras, Journal of Algebra, 378 (2013), p. 64-79.

[5] D. Calaque, A. Căldăraru, J. Tu, On the Lie algebroid of a derived selfintersection, preprint arXiv:1306.5260.

[6] D. Calaque, M. Van Den Bergh, Hochschild cohomology and Atiyah classes, Advances in Math., 224 (2010), no. 5, 1839-1889.

[7] Z. Chen, M. StiÉnon, P. Xu, From Atiyah Classes to Homotopy Leibniz Algebras, preprint arXiv:1204.1075.

[8] D. Grinberg, Poincaré-Birkhoff-Witt type results for inclusions of Lie algebras, Diploma Thesis available at http://www.cip.ifi.lmu.de/ grinberg/ algebra/pbw.pdf.

[9] M. Kapranov, Rozansky-Witten invariants via Atiyah classes, Compositio Math., 115 (1999), no. 1, 71-113.

[10] M. KAPRANov, Free Lie algebroids and the space of paths, Selecta Math. NS, 13 (2007), no. 2, 277-319.

[11] L. Positselski, Two kinds of derived categories, Koszul duality, and comodule-contramodule correspondence, Memoirs of the Amer. Math. Soc., 212 (2011), $133 \mathrm{pp}$.

[12] G.S. Rinehart, Differential forms on general commutative algebras, Trans. Amer. Math. Soc., 108 (1963), 195-222.

[13] P. Xu, Quantum groupoids, Comm. Math. Phys., 216 (2001), no. 3, 539-581.

[14] S. YU, Dolbeault dga of formal neighborhoods and $L_{\infty}$-algebroids, draft available at http://www.math.psu.edu/yu/papers/DolbeaultDGA.pdf.

Manoscritto pervenuto in redazione l'11 Maggio 2012. 
